\title{
Tune It Down to Live It Up? Rapid, Nongenomic Effects of Cortisol on the Human Brain
}

\author{
Florian Strelzyk, ${ }^{1,6}$ Michael Hermes, ${ }^{2}$ Ewald Naumann, ${ }^{1}$ Melly Oitzl, ${ }^{3}$ Christoph Walter, ${ }^{4}$ Hans-Peter Busch, ${ }^{5}$ \\ Steffen Richter, ${ }^{6}$ and Hartmut Schächinger ${ }^{6}$ \\ ${ }^{1}$ Psychophysiological Laboratory of the University of Trier, 54286 Trier, Germany, ${ }^{2}$ Institute of Psychology, University of Heidelberg, 69117 Heidelberg, \\ Germany, ${ }^{3}$ Leiden/Amsterdam Center for Drug Research and Leiden University Medical Center, 2300 RA Leiden, The Netherlands, ${ }^{4}$ Joint Practice for \\ Radiology Dres. Henke/Walter, 54290 Trier, Germany, ${ }^{5}$ Krankenhaus der Barmherzigen Brüder, Department of Radiology, Ultrasound and Nuclear \\ Medicine, 54292 Trier, Germany, and ${ }^{\circ}$ Department of Clinical Physiology, University of Trier, 54286 Trier, Germany
}

The stress hormone cortisol acts on the brain, supporting adaptation and time-adjusted coping processes. Whereas previous research has focused on slow emerging, genomic effects of cortisol, we addressed the rapid, nongenomic cortisol effects on in vivo neuronal activity in humans. Three independent placebo-controlled studies in healthy men were conducted. We observed changes in CNS activity within 15 $\mathrm{min}$ after intravenous administration of a physiological dose of $4 \mathrm{mg}$ of cortisol (hydrocortisone). Two of the studies demonstrated a rapid bilateral thalamic perfusion decrement using continuous arterial spin labeling. The third study revealed rapid, cortisol-induced changes in global signal strength and map dissimilarity of the electroencephalogram. Our data demonstrate that a physiological concentration of cortisol profoundly affects the functioning and perfusion of the human brain in vivo via a rapid, nongenomic mechanism. The changes in neuronal functioning suggest that cortisol acts on the thalamic relay of background as well as on task-specific sensory information, allowing focus and facilitation of adaptation to challenges.

\section{Introduction}

Glucocorticoids (GCs) are adrenal steroid hormones released during stress. They modulate a variety of psychobiological processes to support adaptation and coping with challenging situations. Many studies have focused on how GCs affect the functioning of the CNS via genomic mechanisms. However, even with mounting evidence for the existence of rapid, nongenomic GC mechanisms (for review, see Evanson et al., 2010; Groeneweg et al., 2011) little is known about their in vivo functional effects on the human CNS.

GC secretion originates from neuroendocrinological feedback and feedforward mechanisms of the hypothalamic-pituitaryadrenocortical (HPA) axis, involving control mechanisms of the limbic forebrain (Ulrich-Lai and Herman, 2009). Circulating GCs (mainly cortisol in humans, corticosterone in rodents) affect the entire body, including the CNS, via binding to glucocorticoid and mineralocorticoid receptors. Both receptors act as ligandactivated transcription factors and induce slowly emerging changes in gene transcription (De Kloet et al., 1998) that alter protein expression, structure, and functioning of affected cells.

Received May 9, 2011; revised Sept. 8, 2011; accepted Oct. 7, 2011.

Author contributions: F.S., E.N., M.O., and H.S. designed research; F.S., H.-P.B., and S.R. performed research; F.S., M.H., M.O., and H.-P.B. contributed unpublished reagents/analytic tools; F.S., M.H., E.N., C.W., and H.S. analyzed data; F.S. and H.S. wrote the paper.

This work was supported by the DFG-sponsored International Research Training Group (DFG GRK 1389/1) on Psychoneuroendocrinology of Stress. Further support came from NWO DN-95-420 and NW0 433-09-251.

Correspondence should be addressed to either Florian Strelzyk, or Dr. Hartmut Schächinger, Universität Trier, FBIPsychologie, Universitätsring 15, 54286 Trier, Germany. E-mail: florian@strelzyk.de or schaechi@uni-trier.de.

D01:10.1523/JNEUROSCI.2384-11.2012

Copyright $\odot 2012$ the authors $\quad 0270-6474 / 12 / 320616-10 \$ 15.00 / 0$
However, within seconds to minutes after GC exposure, nongenomic effects of GC occur (Makara and Haller, 2001; Groeneweg et al., 2011). Indeed, it is believed that any rapid effect that occurs $<15-20$ min after GC infusion cannot be mediated by changes in protein expressions (Mikics et al., 2004). That these fast effects originate independently from novo protein synthesis has been demonstrated in an electrophysiological study (Karst et al., 2005).

Our knowledge about rapid, nongenomic GC action in the CNS is scarce and originates primarily from in vitro studies and animal models (Coirini et al., 1983; De Kloet et al., 1984; Mikics et al., 2004; Ferris and Stolberg, 2010), with a few in vivo studies in humans (Kuehl et al., 2010; Lovallo et al., 2010; Richter et al., 2011). In nonhuman models, GCs were reported to rapidly modulate behaviors that originate in the pons, hypothalamus, midbrain, and limbic system. These rapid GC actions involve increased locomotion, food intake, ingestion of carbohydrates, vocalization, aggressive behavior, decreased sexual clasping, memory, and ACTH secretion (Dallman, 2005). Despite their far reaching implications for understanding the rapid adaptation to stress, the neurophysiological correlates of these behavioral effects have not been systematically investigated in humans. In humans, rapid nongenomic effects can only be studied by applying intravenous infusions. Just one previous imaging study (Lovallo et al., 2009) assessed CNS hemodynamic changes after a pharmacological cortisol infusion in humans, but did not report cortisol effects within the first $15 \mathrm{~min}$ after cortisol infusion.

Our aim was to determine whether a cortisol infusion (4 mg of intravenous hydrocortisone) similar to naturally occurring cortisol boosts may exert rapid, and thus putative, nongenomic effects on the 


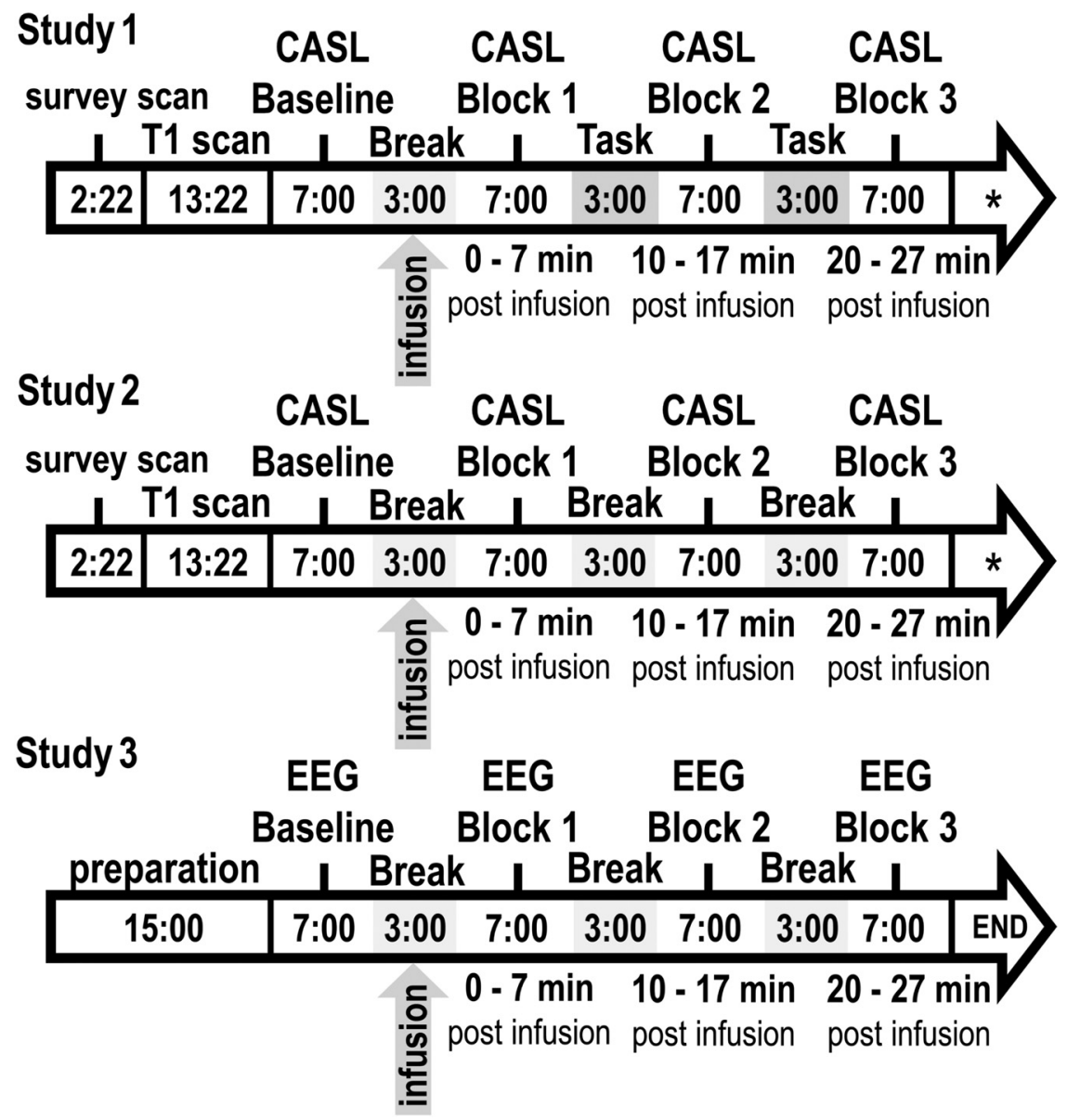

Figure 1. Sequence and duration (in minutes) of experimental procedures during data acquisition (Studies 1, 2, and 3). *The CASL measurements were succeeded by an M0 measurement ( $3 \mathrm{~min}, 25 \mathrm{~s}$ ) and a T2-weighted anatomical measurement ( 2 min, 54 s) to further control for physiological abnormalities. The overall order and duration of the experimental measurement blocks (Baseline, Block 1, Block 2, and Block 3) were identical in all three studies.

human brain. Three independent, placebo-controlled, and doubleblind studies were conducted. Study 1 used regional cerebral blood flow (rCBF) measurements and identified brain regions that responded rapidly to cortisol administration. The main findings were replicated in Study 2. Study 3 was designed to confirm the existence of rapid GC-induced functional correlates in the electroencephalogram (EEG) by assessing the spectral power of spontaneous oscillations and the global markers of signal strength and map dissimilarity. Together, these studies provide tje first neuroimaging evidence for the existence of rapid, nongenomic GC actions in the human CNS.

\section{Materials and Methods}

Hemodynamic measurements: Studies 1 and 2

Participants

Right-handed, psychologically and physiologically healthy, nonsmoking men participated in Study 1 ( 26 men; mean age, 25.54 years; SD, 2.94 years) and Study 2 (nine men; mean age, 23.22 years; SD, 1.86 years). Before MRI measurements, informed consent was obtained and screening interviews determined suitability for MR imaging (beyond establishing MR safety requirements, participants with claustrophobia and discomfort toward medical equipment or procedures were excluded). The studies were approved by the local ethics committee.

\section{Experimental procedure}

In Study 1, participants were measured once in a between-subjects design and were randomly assigned to either $\operatorname{cortisol}(N=13)$ or placebo $(N=$
13) groups and to MRI acquisition time slots that were balanced between the experimental groups. All participants arrived $1 \mathrm{~h}$ before the MRI measurements and a flexible intravenous infusion line was placed into their arm and remained there until the end of the experiment. The $1 \mathrm{~h}$ long waiting period before the start of the experiment was intended to reduce potential carry-over stress effects related to the initial pain induced by placing the intravenous infusion line. Thirty minutes before MRI acquisition, all participants practiced a word discrimination task to familiarize them with a similar task in the scanner. MRI measurements lasted $1 \mathrm{~h}$ and had four experimental resting measurement blocks (each $6 \mathrm{~min}, 50 \mathrm{~s}$ long; referred to as $7 \mathrm{~min}$ from here on) that were intermitted by 3 -min-long breaks (Fig. 1). The infusion of hydrocortisone $(4 \mathrm{mg}$; hydrocortisone is the pharmaceutical term for synthetically produced cortisol with identical binding characteristics) or physiological saline solution (placebo) was administered from outside the scanner room in a double-blind fashion during the first break. During the two remaining breaks, participants were asked to perform the word discrimination task to keep them engaged in the experiment. The task required participants to determine whether prerecorded, audibly presented nouns had either one or two syllables (examples translated into English: e.g., two syllables: "topic"; one syllable: "plan"), by button-presses of either their right or left hand. The nouns were chosen to avoid emotional arousal during the MRI measurements. After the MRI-measurement was finished, the participants were compensated (50€) and informed whether they had received cortisol or placebo.

In Study 2, participants $(N=9)$ were measured twice, 1 week apart and at exactly the same time of day, in a within-subject design. They received cortisol and placebo in a randomized yet balanced order, to control sequence effects. Repeated measurements in the same participants maintained the interpretability and reliability of the ensuing results, while measuring fewer participants (Stevens, 1996). The general experimental procedures were identical to Study 1 (Fig. 1). However, because the perfusion effects in Study 1 may have been (at least in parts) attributable to the task during the break, in Study 2, participants were told that they had been assigned to a waiting control group and they were asked to use all breaks to rest. This resting condition was used to control for possible task-related influences that may have contributed to the results of Study 1. After the second MRI session, participants were compensated (100€) and informed about when they had received cortisol or placebo.

\section{MRI measurements}

rCBF was acquired by applying continuous arterial spin labeling (CASL), which reduces between-subject variability in activation amplitude, improves signal-to-noise ratio in regions with high static susceptibility gradients, and provides superior functional localization compared with BOLD fMRI (Wang et al., 2008). Due to the short decay rate of the endogenous tracer (label relaxes with T1 of arterial blood), CASL measurements may be repeated many times at short intervals. CASL allows the noninvasive quantification of $\mathrm{rCBF}$ in physiological units $(\mathrm{ml} / 100$ $\mathrm{g} / \mathrm{min}$ ) both at rest and during activation.

Data acquisition. Imaging was performed on a $1.5 \mathrm{~T}$ scanner (Intera; Philips Medical Systems) with interleaved label and control images acquisitions, using a single-shot spin echo EPI sequence (Hermes et al., 
2009). Thirteen slices covering the whole brain were acquired (FOV, 230 $\mathrm{mm}$; matrix, $64 \times 64$; slice thickness, $8 \mathrm{~mm}$; $1 \mathrm{~mm}$ gap; bandwidth, 78.4 $\mathrm{kHz}$, flip angle, $90^{\circ}$; TR, $4.125 \mathrm{~ms}$; TE, $42 \mathrm{~ms}$ ) and reconstructed on a $128 \times 128$ matrix. The labeling plane was placed $60 \mathrm{~mm}$ beneath the center of the imaging slices (labeling duration, $2.2 \mathrm{~s}$; labeling amplitude, $35 \mathrm{mg}$; labeling gradient, $0.25 \mathrm{~g} / \mathrm{cm}$; postlabeling delay, $0.8-1.8 \mathrm{~s})$. CBF was measured in four CASL measurement blocks. Each of the four measuring blocks ( $7 \mathrm{~min}$ ) consisted of 46 individual CASL acquisitions.

To familiarize participant to the scanner environment, a T1-weighted sequence (fast field echo; 160 slices; FOV, $256 \times 192 \mathrm{~mm}$; matrix, $256 \times$ 256; slice thickness, $1 \mathrm{~mm}$; TR, $11.9 \mathrm{~ms}$; TE, $3.3 \mathrm{~ms}$; duration, $13 \mathrm{~min}$, $22 \mathrm{~s}$ ) was acquired before the CASL measurements. After CASL, a series of $46 \mathrm{M} 0$-images was acquired to determine the equilibrium magnetization (duration, $3 \mathrm{~min}, 25 \mathrm{~s}$ ).

Data processing. Offline data processing of CASL and T1 images was performed with the Statistical Parametric Mapping Software (SPM8; Welcome Department of Imaging Neuroscience, London UK; implemented in MATLAB 2008; MathWorks), using the procedures described by Hermes and colleagues (2007). We averaged the label and nonlabel images separately for each of the four CASL acquisition blocks. The M0 images were averaged separately. The label, nonlabel, and M0 averages were used to quantify CBF following forms as described by Alsop and Detre (1996); a blood-brain partition coefficient of water of $\lambda=0.98$ was used for gray matter quantification (Herscovitch and Raichle, 1985; Roberts et al., 1996).

After quantification, T1 images were segmented and converted into dichotomous gray matter masks. These gray matter masks were multiplied with the CBF images, which resulted in CBF maps for gray matter to solely analyze gray matter CBF. In Study 1, primary ROIs consisted of regions of the limbic lobe and the subcortical gray matter, because these structures are likely involved in fast cognitive stress effects (Sutanto et al., 1988; Dedovic et al., 2009) and have been reported to show cortisolinduced effects (though not rapid) under low cognitive load (Lovallo et al., 2010). However, since GC binding sites are distributed throughout the entire brain (Sánchez et al., 2000), exploratory ROIs consisted of all regions in the frontal, temporal, parietal, and occipital lobes, and central region. These ROIs have not been demonstrated to show cortisolinduced effects during low cognitive load in humans, but can be speculated to do so. Primary and exploratory ROIs together consisted of 45 regions, as defined based on published templates (Tzourio-Mazoyer et al., 2002), and were used to create dichotomous ROI masks. ROIs were grouped by their superordinate lobe or area location to classify them according to their anatomical relationship [for region labels, see MARINA toolbox (Walter et al., 2003)]. Finally, the ROI masks were multiplied with the gray matter $\mathrm{CBF}$ images and the mean $\mathrm{rCBF}$ in each $\mathrm{ROI}$ was calculated intraindividually.

In Study 2, the ROI analysis was hypotheses driven to replicate the main findings of Study 1 . Therefore, the only ROIs examined were the thalamus and caudate nucleus (replication ROIs).

\section{Basal HPA axis activity}

Measurements of basal HPA axis activity were obtained via the cortisol awakening response (Pruessner et al., 1997) on the day of the MRI measurement for Study 1 and on each of the $2 \mathrm{~d}$ of the MRI measurements for Study 2 . The cortisol awakening response was acquired via four salivary cortisol measurements $0,30,45$, and 60 min after awakening (Wilhelm et al., 2007). Basal HPA axis activation was used to check for systematic differences between cortisol and placebo groups or conditions. Salivary cortisol was analyzed via an immunoassay with fluorescence detection (Dressendörfer et al., 1992).

\section{Manipulation check}

To check whether the pharmacological manipulation succeeded, measurements of acute HPA axis activity (i.e., cortisol levels) were obtained through three saliva samples per session. Participants provided their first sample upon arrival, the second before entering the MRI scanning room, and the third after the last MRI sequence. Salivary cortisol was analyzed via an immunoassay with fluorescence detection (Dressendörfer et al., 1992).

\section{Statistical analyses}

Basal HPA axis activity. Cortisol awakening responses were quantified for each measurement day by calculating the area under the curve relative to ground (AUCg), as described by Pruessner and colleagues (2003). Quantification resulted in one AUCg score per participant for Study 1, and two AUCg scores per participant for Study 2. AUCg values of cortisol and placebo measurements for each study were submitted into an unpaired (Study 1) or paired (Study 2) $t$ test.

Manipulation check. For Studies 1 and 2, the pharmacological manipulation was assessed via ANOVA with the intervention (cortisol, placebo) and measurement time (arrival, before MRI, after MRI) as mixed between-subject, or completely repeated-measures factorial design, respectively.

ROI analysis. A ROI-based approach decreases the likelihood of falsepositive results during the brain-wide result exploration.

In Study 1, we assessed individual rCBF averages of each ROI by performing an analysis of covariance (ANCOVA) to avoid multiple $t$ tests between the ROIs, control for the compounding error rates, and increase the statistical power of the analyses. We intraindividually subtracted baseline $\mathrm{rCBF}(-7-0 \mathrm{~min}$ before cortisol infusion) from the remaining three CASL measurement blocks to obtain the absolute change of $\mathrm{rCBF}$ relative to baseline of each ROI. This accounted for naturally occurring personal baseline differences between participants, which, in our sample, reached from $61.12-91.44 \mathrm{ml} / 100 \mathrm{~g} / \mathrm{min}$ in global gray matter CBF. Baseline-corrected rCBF values were used for all further ANCOVA.

A separate ANCOVA was performed for each anatomical lobe or area of the primary and exploratory ROIs, entering the factors intervention (cortisol, placebo), measurement block (0-7 min, 10-17 min, 20-27 min postinfusion), region (according to ROIs), and hemisphere (left, right). The ANCOVAs contained the individual CASL baseline perfusion of the gray matter CBF images as a covariate to reduce unexplained statistical variance (i.e., error variance) that can be attributed to differences in baseline perfusion. The CASL baseline covariate led to no significant effects with the intervention (cortisol, placebo) in any of the performed ANCOVAs. Therefore, the covariate only cleared up unexplained variance and did not carry significant intervention effect variance in itself. Thus, the effects of intervention (cortisol, placebo) are unrelated to preinfusion effects of the baseline that are due to naturally occurring interindividual differences.

A Huynh-Feldt correction of the degrees of freedom was performed whenever appropriate. Significant effects $(\alpha<0.05)$ were further analyzed using Dunn's multiple comparison procedure (Dunn, 1961) as post hoc analyses and the critical differences $(\psi)$ for $p<0.05, p<0.01$, and the number of comparisons $(C)$ are stated. The presentation of results will be limited to significant $(p<0.05)$ intervention-related effects and according degrees of freedom $\left(\mathrm{df}_{\text {effect }} ; \mathrm{df}_{\text {error }}\right)$, and the effect size measure partial $\eta^{2}$ (Cohen, 1988) are reported.

Voxel-based exploration. In Study 1, a voxel-based result exploration was set up within the framework of the general linear model (Friston et al., 1995), which is used in SPM8. Voxel-based results are supposed to solely provide a descriptive overview of the spatial effect distribution to exclude faulty interpretations of the ROI analyses. Therefore, the significance threshold was set to a liberal criterion of $p<0.01$ (uncorrected). Thus, the voxel-based description was not used for statistical interpretations. The mean (unsegmented and smoothed) CBF images were used in random effects full factorial models. We calculated statistical parametric maps (SPMs) for $\mathrm{CBF}$ changes for the interaction intervention $\times$ measurement. The interaction contrasts were set up to account for interindividual differences in the CASL baseline by subtracting the between-group baseline effect from the three postintervention CASL measurement blocks. To minimize the contribution of extracerebral voxels, we used an absolute CBF threshold of $5 \mathrm{ml} / 100 \mathrm{~g} / \mathrm{min}$.

In Study 2, due to the a priori hypothesis, raw perfusion values of the bilateral thalamus and caudate nucleus ROIs were assessed via one-tailed, paired Student's $t$ tests of the baseline and the remaining three CASL measurement blocks [applying Bonferroni-Holm (Holm, 1979) procedure to correct for multiple tests]. No baseline correction was necessary since the repeated measurements of the same participants avoided significant baseline differences between the cortisol and placebo conditions. 
The lack of significant baseline differences matches earlier findings that showed that resting CASL measurements, as well as variations in CASL perfusion measurements, of the same participants and acquired several weeks apart are a stable personal trait and remain relatively unchanged (Hermes et al., 2007).

Correlation analyses. To assess the impact of intervention-induced cortisol changes on the CBF, Pearson product-moment correlation coefficients were obtained between the individual baseline-corrected perfusion values of the bilateral thalamus of the first measurement block (cortisol infusion showed its statistical effect in both studies) and the acute cortisol effect (acute saliva cortisol level after MRI minus acute cortisol level before MRI) of Study 1 and Study 2.

All statistical analyses were performed with SPSS for Windows (Version 17 , SPSS).

\section{Electroencephalographic measurements: Study 3 Participants}

Fourteen right-handed healthy men (mean age, 24.50 years; SD, 2.14 years) participated in the experiment. All screening and exclusion criteria were identical to Studies 1 and 2.

\section{Experimental procedure}

As in Study 2, participants were measured twice, 1 week apart and at exactly the same time of day, in a within-subject design. The general experimental procedures and intervention ( $4 \mathrm{mg}$ of cortisol) were identical to Studies 1 and 2 (Fig. 1), however, participants were seated in a psychophysiological laboratory and prepared for EEG measurements. During the EEG resting measurements, participants kept their eyes open or closed in an intraindividually and interindividually balanced order that alternated between and within the individual EEG measurement blocks ( per condition, starting blocks with open-closed-open-closed or closed-open-closed-open). As in Study 2, during the second break, participants were told that they had been randomly assigned to a waiting control group and they were asked to use all following breaks to rest. As in Study 2, participants were instructed to provide saliva samples after awakening to assess the basal HPA axis response.

\section{EEG measurement, preprocessing, and analyses}

EEG was recorded continuously by $32 \mathrm{Ag}-\mathrm{AgCl}$ pin-type active electrodes (Brain-amp; Brain Products). The electrodes $[\mathrm{FP}(1,2), \mathrm{F}(3, \mathrm{z}, 4), \mathrm{FC}(7,2$, $4,8), \mathrm{T}(7,1,2,8), \mathrm{C}(3, \mathrm{z}, 4), \mathrm{CP}(5,1,2,6), \mathrm{P}(7,3, \mathrm{z}, 4,8), \mathrm{O}(1, \mathrm{z}, 2), \mathrm{A}(1$, $2)$ ] were mounted according to the 10-20 system (Chatrian et al., 1988) and referenced to FCz. Impedances of the EEG electrodes were $<10$ $\mathrm{kOhm}$. The high-pass filter was set to $0.0159 \mathrm{~Hz}$ ( $24 \mathrm{~dB} /$ octave roll-off), and the low-pass filter was set to $250 \mathrm{~Hz}$. The signals were digitized at $1000 \mathrm{~Hz}$ and stored for offline analysis.

The EEG was re-referenced to mathematically linked mastoids, refiltered (bandpass: $1-40 \mathrm{~Hz} ; 24 \mathrm{db}$ ) to minimize drifts and noise that were present in some data channels, and resampled to $250 \mathrm{~Hz}$. Continuous EEG recordings were segmented into nonoverlapping $4 \mathrm{~s}$ epochs for each measurement block and visually inspected. Epochs with nonstereotyped artifacts (e.g., electrode cable movements, swallowing, etc.) were rejected from further analysis (mean across blocks for cortisol and placebo: 5.2 and 5.7 epochs, respectively; range across blocks for cortisol and placebo: 17 and 20 epochs, respectively), whereas epochs containing repeatedly occurring, stereotyped ocular and heart-beat-related artifacts were corrected using infomax independent component analysis (ICA) (Bell and Sejnowski, 1995; Makeig et al., 1996; Jung et al., 2000). Following this procedure, ocular and heart beat artifact-corrected data were created via back-projection (average removed components per participant: cortisol, 5.82; placebo, 5.58).

Studies 1 and 2 revealed cortisol-induced effects on the thalamus, which implies fundamental and global changes on EEG. Therefore, effects of cortisol infusion on global EEG functioning were assessed using global field power (GFP; spatial standard deviation), which quantifies global signal strength, and global map dissimilarity (GMD; the spatial square root of the mean of the squared differences between two maps), which measures the dissimilarity between topographical scalp maps
(Lehmann et al., 1987; Koenig and Gianotti, 2009). Scalp potential fields with pronounced peaks and steep gradients result in high GFP (i.e., high signal strength), while GFP is low in maps with flat appearance and shallow gradients. GMD is 0 when two maps are equal (i.e., no dissimilarity) and maximally reaches 2 when the maps have the same topography with reversed polarity. GFP and GMD were obtained from each data point or consecutively paired data points (for GMD) of the artifactcorrected epochs and averaged separately for each measurement block, resulting in a single value per block and participant.

In measurement blocks in which the signal is generally very hilly due to large amplitudes, the GFP will, on average, be higher than in blocks that contain shallower, low amplitude signals. An increase in the GMD average of an EEG measurement block implies that the containing topographical maps have changed much more often or to a much larger extent, whereas a GMD decrease implies that the neuronal processing during this measurement block did not alter as often or as much. Such statements are valuable to establish whether or not cortisol induces any measurable effect on neuronal functioning.

Spectral power density $\left[\mu \mathrm{V}^{2} / \mathrm{Hz}\right]$ was calculated by applying fast Fourier transformation (FFT) to the artifact-corrected epochs of $4 \mathrm{~s}$, resulting in a maximum resolution of $0.25 \mathrm{~Hz}$. All epochs overlapped ( $10 \%$ at each end) to compensate the differential weighting of data points (Dumermuth and Molinari, 1987) and were extracted through a Hanning window that was specified to diminish the signal $10 \%$ at each end to prevent spurious estimates of spectral power (Bloomfield, 1976). The resulting spectral epochs were averaged and ln-transformed (Gasser et al., 1982), and spectral power density for the frequency bands $4-8 \mathrm{~Hz}$, $8-12 \mathrm{~Hz}$, and $12-30 \mathrm{~Hz}$ were created for each electrode. Electrodes at the location F, FC, C, CP, P, and O were averaged according to coronal rows on the scalp to include them as the topographical factor caudality (frontal, frontocentral, central, centroparietal, parietal, occipital) during statistical analyses.

The postinfusion measurements of spectral power density, GFP and GMD (0-7 $\mathrm{min}, 10-17 \mathrm{~min}, 20-27 \mathrm{~min})$, were baseline-corrected intraindividually by subtracting the preinfusion measurement $(-7-0 \mathrm{~min}$ before infusion); the baseline corrected values were used for ANOVA. The artifact screening, re-referencing, and ICA were performed with Brain Vision Analyzer 2.0 (BrainProducts); FFT (applying pwelch function) and GFP and GMD analyses were performed in MATLAB.

\section{Basal HPA axis activity}

The assessment of basal HPA axis activity was identical to Study 2.

\section{Manipulation check}

The assessment of acute HPA axis activity was identical to Study 2.

\section{Statistical analyses}

Analysis of the pharmacological manipulation was identical to Study 2.

Global EEG measures (GMD, GFP) were submitted each to a separate ANOVA, entering the factors intervention (cortisol, placebo) and measurement block ( $0-7 \mathrm{~min}, 10-17 \mathrm{~min}, 20-27 \mathrm{~min}$ postinfusion) as repeated measures. Spectral power density measures were submitted to an ANOVA, entering the factors intervention (cortisol, placebo), measurement block (0-7 min, 10-17 min, 20-27 min postinfusion), caudality (frontal, frontocentral, central, centroparietal, parietal, occipital) and frequency band $(4-8 \mathrm{~Hz}, 8-12 \mathrm{~Hz}, 12-30 \mathrm{~Hz})$ as repeated measures. Statistical criteria are identical to Studies 1 and 2.

Correlation analysis. To assess the impact of the intervention-induced cortisol change on global EEG markers, Pearson product-moment correlation coefficients were obtained between the individual baselinecorrected signal strengths (GFP) averaged across all three experimental measurement blocks (since cortisol induced changes across all blocks) and the acute cortisol effect (acute saliva cortisol level after MRI minus acute cortisol level before MRI). An identical analysis was performed for map dissimilarity (GMD) parameters. 
Table 1. ANCOVA results for all anatomical groups of Study 1, separated according to primary and exploratory ROIs

\begin{tabular}{|c|c|c|c|c|c|c|c|}
\hline & \multicolumn{2}{|l|}{ Primary ROIs } & \multicolumn{5}{|c|}{ Exploratory ROls } \\
\hline & Limbic lobe & Subcortical gray matter & Frontal lobe & Temporal lobe & Parietal lobe & Occipital lobe & Central region \\
\hline \multicolumn{8}{|l|}{ Intervention $\times$ hemisphere } \\
\hline df & $1 ; 23$ & $1 ; 23$ & $1 ; 23$ & $1 ; 23$ & $1 ; 23$ & $1 ; 23$ & $1 ; 23$ \\
\hline$F$ & 1.805 & 2.203 & 5.914 & 0.010 & 0.109 & 6.976 & 0.532 \\
\hline$p$ & 0.192 & 0.151 & 0.023 & 0.920 & 0.744 & 0.015 & 0.473 \\
\hline$\eta_{\text {part }}^{2}$ & & & 0.205 & & & 0.233 & \\
\hline \multicolumn{8}{|l|}{ Intervention $\times$ measurement block $\times$ region } \\
\hline $\mathrm{df}$ & $12 ; 276$ & $10 ; 230$ & $24 ; 552$ & $6 ; 138$ & $8 ; 184$ & $12 ; 276$ & $4 ; 92$ \\
\hline$F$ & 1.309 & 2.447 & 1.223 & 0.348 & 0.528 & 0.890 & 0.349 \\
\hline$p$ & 0.228 & 0.020 & 0.276 & 0.859 & 0.782 & 0.536 & 0.788 \\
\hline$\eta_{\text {part }}^{2}$ & & 0.096 & & & & & \\
\hline \multicolumn{8}{|l|}{ Intervention $\times$ region $\times$ hemisphere } \\
\hline $\mathrm{df}$ & $6 ; 138$ & $5 ; 115$ & $12 ; 276$ & $3 ; 69$ & $4 ; 92$ & $6 ; 138$ & $2 ; 46$ \\
\hline$F$ & 0.678 & 0.313 & 1.409 & 0.991 & 1.861 & 3.121 & 0.346 \\
\hline$p$ & 0.606 & 0.831 & 0.170 & 0.374 & 0.124 & 0.017 & 0.709 \\
\hline$\eta_{\text {part }}^{2}$ & & & & & & 0.119 & \\
\hline \multicolumn{8}{|l|}{ Intervention $\times$ measurement block $\times$ hemisphere } \\
\hline df & $2 ; 46$ & $2 ; 46$ & $2 ; 46$ & $2 ; 46$ & $2 ; 46$ & $2 ; 46$ & $2 ; 46$ \\
\hline$F$ & 1.926 & 0.277 & 0.261 & 0.073 & 4.158 & 0.578 & 0.241 \\
\hline & 0.157 & 0.760 & 0.771 & 0.930 & 0.022 & 0.565 & 0.787 \\
\hline$\eta_{\text {part }}^{2}$ & & & & & 0.153 & & \\
\hline
\end{tabular}

Significant effects are found in the subcortical gray matter, frontal lobe, parietal lobe, and occipital lobe (bold). df, Degrees of freedom; $F, F$ values; $p, p$ values; $\eta_{\text {part }}^{2}$, partial $\eta^{2}$.

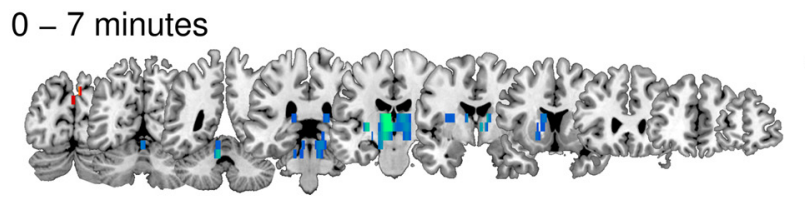

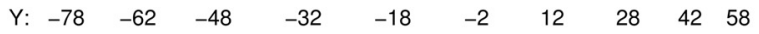
10 - 17 minutes

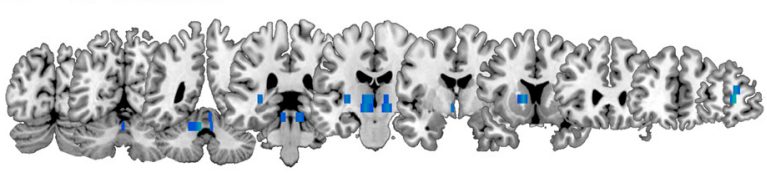

$20-27$ minutes

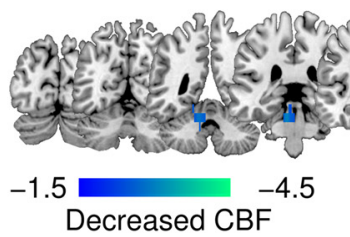

Decreased CBF

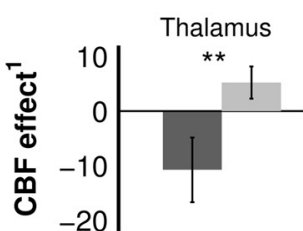

Caudate Nucleus

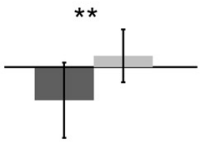

1 in $\mathrm{ml} / 100 \mathrm{~g} / \mathrm{min}$
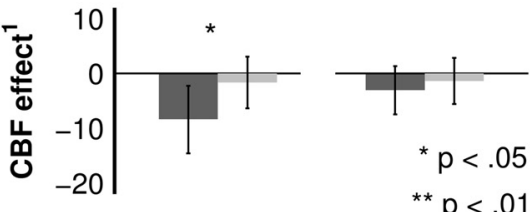

${ }^{\star *} \mathrm{p}<.01$

$95 \% \mathrm{Cl}$
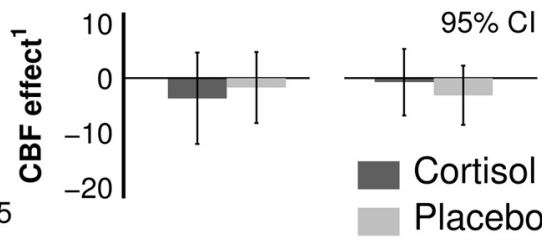

Figure 2. Cortisol-induced rCBF changes (Study 1). Left, Statistical parametric maps for CBF changes for the interaction intervention $\times$ measurement (baseline corrected contrasts, cortisol vs placebo; $p<0.01$, uncorrected) provide a descriptive overview of the spatial effect distribution and justify the interpretations of the ROl analyses. Locally distinct bilateral perfusion decrements in the thalamus and caudate nucleus (baseline corrected) after the intravenous infusion of cortisol ( $4 \mathrm{mg}$ ) for each measurement block (time, minutes after infusion). Right, Bilaterally averaged and baseline-corrected rCBF for significant ROI comparisons for all postinfusion measurement blocks [95\% confidence interval CI)].

\section{Results}

Hemodynamic measurements: Studies 1 and 2

Basal HPA axis activity

No significant differences were found between the cortisol and placebo groups of Study $1\left(t_{(24)}=-1.11 ; p=0.27\right)$ or between the cortisol and placebo conditions of Study $2\left(t_{(8)}<1\right)$.

Manipulation check

The cortisol intervention was successful in Study $1\left(F_{(2,48)}=5.066\right.$, $\left.p=0.015, \eta_{\text {part }}^{2}=0.174\right)$ and Study $2\left(F_{(2,16)}=3.830, p=0.044\right.$, $\left.\eta_{\text {part }}^{2}=0.324\right)$, with significant salivary cortisol increases directly after the MRI measurements in the cortisol but not in the placebo group (significant increases, for cortisol conditions: Study 1, 2.6 nmol/l; Study 2, $4.1 \mathrm{nmol} / \mathrm{l}$ ). In addition, there were no significant differences in the salivary cortisol levels between cortisol and placebo interventions immediately before MRI measurement in both studies.
Study 1: primary ROIs

Cortisol infusion significantly affected $\mathrm{rCBF}$ in regions of the subcortical gray matter, which is expressed in a significant threeway interaction $\left(F_{(10,230)}=2.447, p=0.020, \eta_{\text {part }}^{2}=0.096\right)$ between intervention, measurement block, and region (Table 1). Post hoc analyses $\left(\psi_{5 \%}=7.07 \mathrm{ml} / 100 \mathrm{~g} / \mathrm{min} ; \psi_{1 \%}=8.16 \mathrm{ml} / 100\right.$ $\mathrm{g} / \mathrm{min}$; $C=18$ ) further decomposed this effect as a significant bilateral perfusion decrease of $15.92 \mathrm{ml} / 100 \mathrm{~g} / \mathrm{min}$ in the thalamus during the first $7 \mathrm{~min}$ after cortisol infusion compared with placebo (Fig. 2). This effect remained in the second $(7.56 \mathrm{ml} / 100$ $\mathrm{g} / \mathrm{min}$ ) but disappeared in the third measurement block, in which the thalamic perfusion values receded back baseline levels. Post hoc analyses further revealed a significant bilateral perfusion decrease of $8.61 \mathrm{ml} / 100 \mathrm{~g} / \mathrm{min}$ in the caudate nucleus during the first $7 \mathrm{~min}$ after the cortisol infusion compared with placebo (Fig. 
Table 2. Effect descriptions for exploratory ROIs (by anatomical group) in Study 1, including the critical differences used to determine statistical significance

\begin{tabular}{|c|c|c|c|c|}
\hline & \multicolumn{3}{|c|}{ Critical difference } & \multirow[b]{2}{*}{ Effect description } \\
\hline & $\psi_{5 \%}$ & $\psi_{1 \%}$ & $C$ & \\
\hline Frontal lobe $e^{b}$ & $1.39^{a}$ & $1.74^{a}$ & 4 & Significant perfusion decrease of $1.66^{a}$ in the right frontal hemisphere of the cortisol group \\
\hline Occipital lobe $e^{b, d}$ & $0.98^{a}$ & $1.22^{a}$ & 4 & $\begin{array}{l}\text { Significant perfusion increase in the right }\left(2.97^{a}\right) \text { and left }\left(1.34^{a}\right) \text { hemispheres of the cortisol group } \\
\text { Middle occipital lobe (lateral surface): Significant effect in the left }\left(4.61^{a}\right) \text {, but not the right }\left(2.70^{a}\right) \text {, hemisphere } \\
\text { Inferior occipital gyrus (lateral surface): Significant effect in the right }\left(3.79^{a}\right) \text {, but not the left }\left(3.33^{a}\right) \text {, hemisphere }\end{array}$ \\
\hline Occipital lobe $e^{b, e}$ & $3.60^{a}$ & $4.21^{a}$ & 14 & $\begin{array}{l}\text { Cuneus (medial and inferior surface): Significant effect in the right }\left(5.85^{a}\right) \text {, but not the left }\left(1.09^{a}\right) \text {, hemisphere } \\
0-7 \text { min: Significant perfusion increase of } 1.84^{a} \text { in the cortisol relative to the placebo group in the right but not the left hemisphere } \\
10-17 \text { min: No significant change in cortisol and placebo groups }\end{array}$ \\
\hline Parietal lobe ${ }^{c}$ & $1.57^{a}$ & $1.91^{a}$ & 6 & $20-27 \mathrm{~min}$ : Significant perfusion increase in the left $\left(4.64^{a}\right)$ and right $\left(2.37^{a}\right)$ hemispheres of the cortisol relative to the placebo group \\
\hline
\end{tabular}

${ }^{a}$ Values are in milliliters per $100 \mathrm{~g}$ per minute ( $\left.\mathrm{ml} / 100 \mathrm{~g} / \mathrm{min}\right) .{ }^{b}$ Lack of significant higher-order interactions concerning measurement block (Table 1 ) signifies that the perfusion differences in the frontal and occipital lobes stretch across all postintervention CASL measurement blocks. This interaction can be attributed to the different effect of intervention on the hemispheres and between the CASL measurement blocks. ${ }^{d}$ Intervention $\times$ hemisphere. ${ }^{e}$ Intervention $\times$ region $\times$ hemisphere.

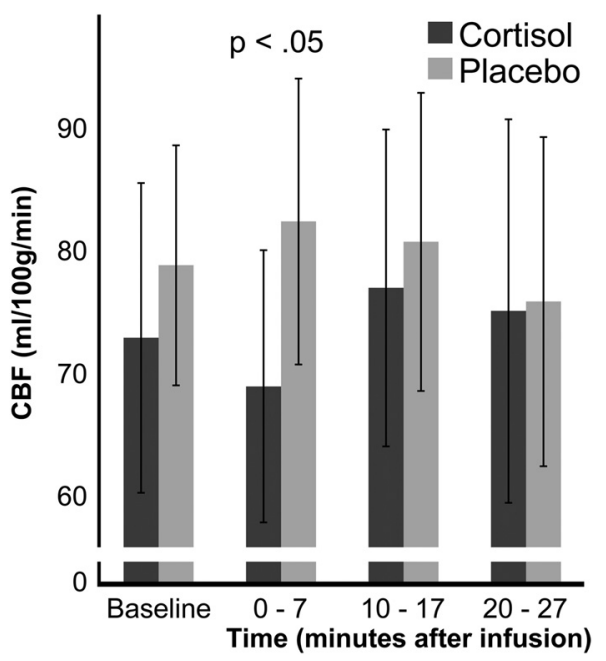

Figure 3. Cortisol-induced rCBF changes in the bilateral thalamus in Study 2. Cortisol induced a bilateral perfusion decrease in the thalamus during the first measurement block $(0-7$ $\mathrm{min}$ ) after the infusion ( $95 \%$ confidence interval). This result replicates the thalamic perfusion decrease during the first measurement block of Study 1.

2). No other significant intervention-related effects were found for the remaining subcortical regions.

Study 1: exploratory ROIs

Three further analyses revealed a significant effect (Table 1) that showed intervention-related effects in the frontal, parietal, and occipital lobes. However, the presentation of these results is limited to a description in Table 2, because the replication in Study 2 was only optimized to replicate the findings of the primary ROIs, which limited the number of ROI comparisons.

Study 2: replication ROIs

Cortisol infusion significantly decreased $\left(t_{(8)}=-3.329 ; p=\right.$ 0.005 ) the bilateral perfusion of the thalamus by $13.45 \mathrm{ml} / 100$ g/min during the first 7 min (Fig. 3) compared with placebo (Table 3). No significant differences (all ps $>0.05$ ) between the cortisol and placebo conditions were found in the baseline measurement and no significant effects of the cortisol infusion were found during the second and third measurement blocks. The caudate nucleus also showed no significant effect.

Studies 1 and 2: correlation analysis

Increases in the cortisol levels were significantly related to decreases in bilateral thalamic perfusion both in Study $1(r=-0.43$; $p=0.03)$ and Study $2(r=-0.46 ; p=0.05)$, as displayed in Figure 5, $A$ and $B$.
Table 3. Result summary of the hypotheses-driven planned comparisons ( $t$ test) for the bilateral thalamus and caudate nucleus (Study 2), with overview of required critical $p$ values

\begin{tabular}{llllll}
\hline ROI & $\begin{array}{l}\text { Cortisol }- \\
\text { placebo }\end{array}$ & $t$ & df & $p$ & $\begin{array}{c}p^{a} \text { for } \\
\alpha<5 \%\end{array}$ \\
\hline $\begin{array}{l}\text { Thalamus, left and right } \\
\quad \text { Baseline }\end{array}$ & -3.700 & -0.842 & 8 & 0.212 & 0.0100 \\
$0-7$ min & -13.453 & -3.329 & 8 & $0.005^{*}$ & 0.0063 \\
$10-17$ min & -3.760 & -0.753 & 8 & 0.236 & 0.0167 \\
$20-27$ min & -0.768 & -0.110 & 8 & 0.457 & 0.0500 \\
Caudate nucleus, left and right & & & & & \\
$\quad$ Baseline & -5.990 & -1.469 & 8 & 0.090 & 0.0083 \\
$0-7$ min & -6.763 & -1.625 & 8 & 0.071 & 0.0071 \\
$10-17$ min & -4.090 & -0.850 & 8 & 0.210 & 0.0125 \\
$20-27$ min & -0.459 & -0.125 & 8 & 0.451 & 0.0250 \\
\hline${ }^{a}$ Critical $p$ value of Bonferoni-Holm correction for eight Student's $t$ tests. * ${ }^{\circ}$ Significant for $p<0.05$, corrected.
\end{tabular}

Electroencephalographic measurements: Study 3

Basal HPA axis activity

No significant differences were found between the cortisol and placebo conditions of Study $3\left(t_{(14)}<1\right)$.

Manipulation check

After inspections of the salivary cortisol measurements, a descriptive increase in the cortisol level after the MRI measurement was observed in 13 of 14 participants. In these 13 participants, the intervention was successful $\left(F_{(2,24)}=9.064, p=0.001, \eta^{2}\right.$ part $=$ 0.411 ) and these were entered into EEG analyses. In these participants, salivary cortisol significantly increased directly after the EEG measurements in the cortisol but not in the placebo condition (significant mean increase in cortisol condition: $3.7 \mathrm{nmol} / \mathrm{l}$ ). Additionally, there was no significant difference in the salivary cortisol levels between the cortisol and placebo conditions immediately before MRI measurement.

Signal strength, spectral power, map dissimilarity

Cortisol infusion significantly decreased signal strength $\left(F_{(1,12)}=\right.$ 41.009, $p<0.001, \eta^{2}$ part $\left.=0.774\right)$ and spectral power density $\left(F_{(1,12)}=18.215, p=0.001, \eta_{\text {part }}^{2}=0.603\right)$ in all postinfusion measurements (Fig. $4 A$ ). Since spectral power is mathematically linked to signal strength, the decrease in GFP (cortisol, -0.701 ; placebo, 0.438 ) is reflected in the spectral power decrease across all bands. Map dissimilarity significantly decreased after infusion in the cortisol compared with the placebo condition (Fig. 4B) across all measurement blocks $\left(F_{(1,12)}=6.532, p=0.025, \eta^{2}\right.$ part $\left.=0.352\right)$. This indicates a decreased topographic dissimilarity in the cortisol compared with the placebo condition. 


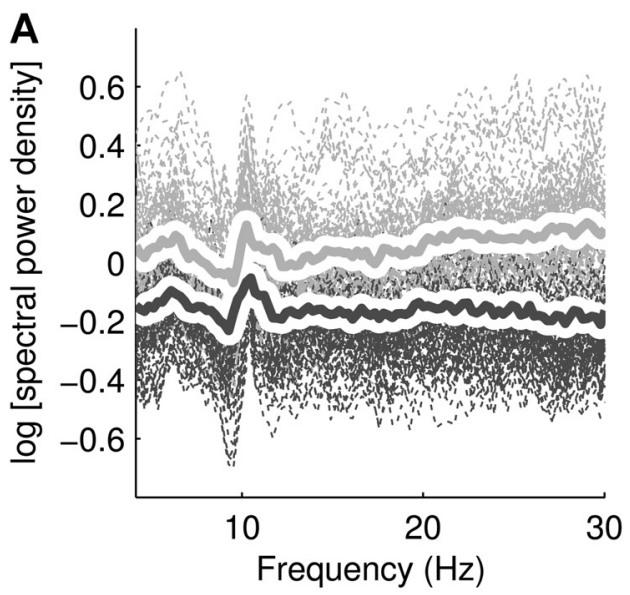

B

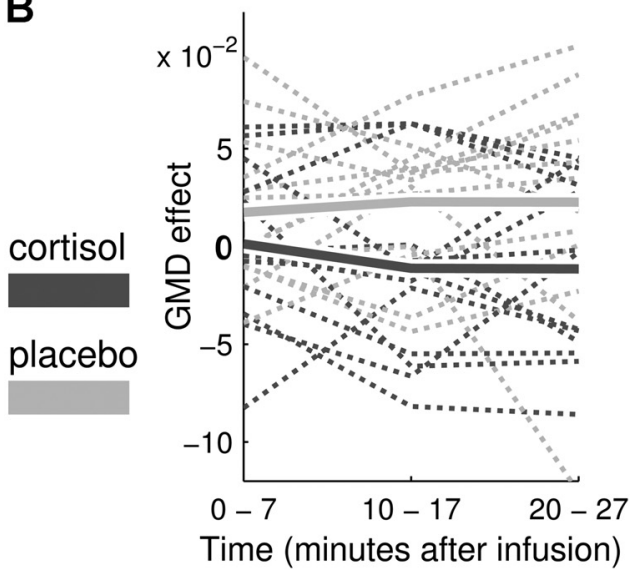

Figure 4. Cortisol-induced changes in global EEG markers (Study 3). $\boldsymbol{A}$, In-transformed and baseline-corrected FFT power spectra for all postinfusion measurement blocks, electrodes, and participants (dashed lines), with the average for cortisol (solid dark gray line) and placebo (solid light gray line) conditions. The cortisol-induced decrease in spectral power stretches across all frequency bins $(4-30 \mathrm{~Hz})$. B, Baseline-corrected GMD for all postinfusion measurement blocks (0-7 min, 10-17 min, 20-27 min) displayed for each participant (dashed lines) and averaged for cortisol (solid dark gray line) and placebo (solid light gray line) conditions.

\section{Correlation analysis}

Increases in the cortisol levels were significantly related to decreases in signal strength (and vice versa) $(r=-0.42 ; p=0.02)$, as displayed in Figure 5C. Map dissimilarity, however, showed no significant correlation with changes in cortisol levels $(r=$ $-0.202 ; p=0.302)$.

\section{Discussion}

This report provides coherent evidence for rapid, large scale effects of GCs on the functioning of the CNS in humans. An infusion of cortisol (hydrocortisone) in the physiological range of endogenous cortisol levels under stress exerts effects on the human brain within $15 \mathrm{~min}$. Using continuous arterial spin labeling as a MRI sequence, Studies 1 and 2 identified rapid bilateral decrement of thalamic perfusion. Study 3 indicated the existence of global and functionally relevant changes in the EEG.

Our studies were specifically designed to explore rapid, putative nongenomic CNS effects of cortisol. The relative modest concentration of $4 \mathrm{mg}$ of cortisol is within the physiological range of endogenous cortisol secretion and was not noticed by our participants (for a discussion of plausible ranges, see Richter et al., 2011). To specifically address the GC component of a stress response, we set up the experiments to avoid mental stress, autonomic nervous system acti-
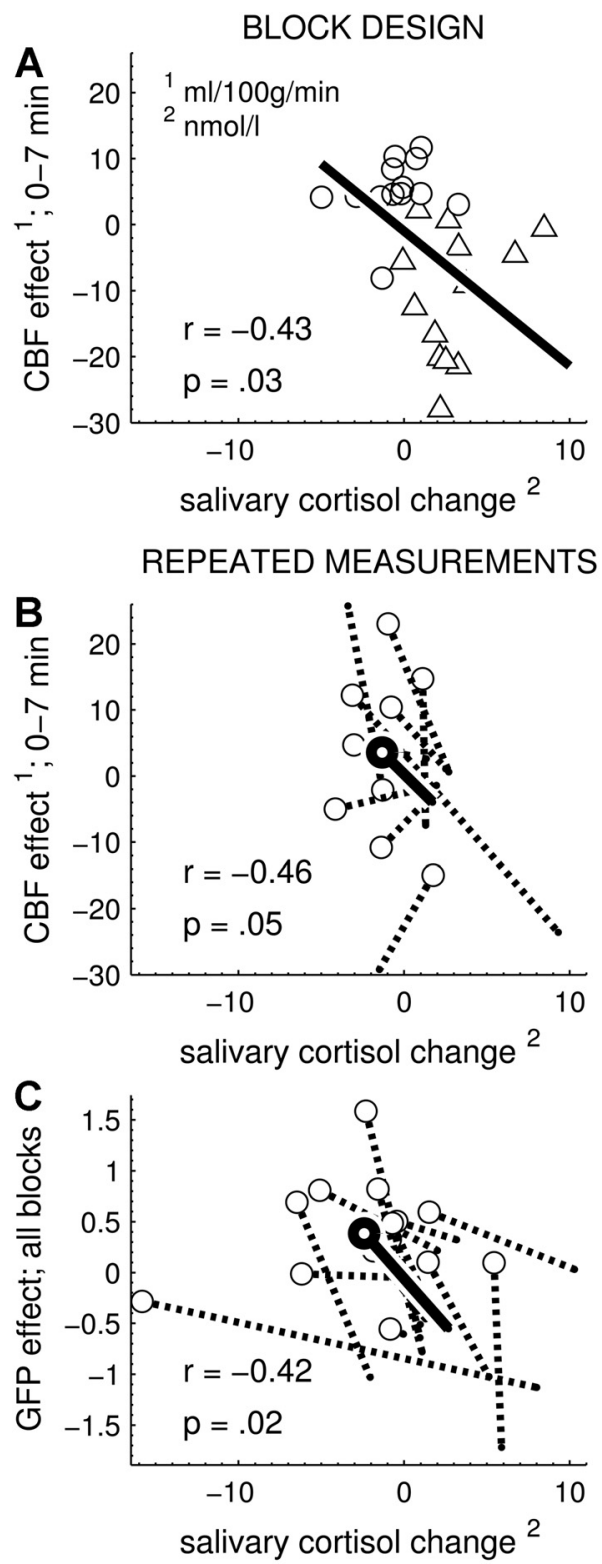

$\begin{array}{llll}\text { cortisol } & \Delta & \text { placebo } & 0 \\ \text { cortisol effect } & =-- & \text { vector average }\end{array}$

Figure 5. Significant correlations between dependent variables and salivary cortisol changes. A, Study 1. Baseline-corrected thalamic blood flow of the first measurement block (ordinate) is negatively correlated with the change in salivary cortisol (abscissa, cortisol levels after MRI minus cortisol levels before MRI). This correlation is plotted across all participants, since cortisol and placebo groups contained different participants. B, Study 2. Baselinecorrected thalamic blood flow of the first measurement block is also negatively correlated with the change in salivary cortisol. This correlation is plotted as individual effect vectors because the cortisol and placebo conditions contained the same participants (repeated measurement study). C, Study 3. Baseline-corrected GFP values (ordinate) were averaged across the three postinfusion measurement blocks (because the effect of intervention was significant across all blocks) and showed a negative correlation with the salivary cortisol change (abscissa, cortisol levels after EEG minus cortisol levels before EEG).

vation, and arousal. Therefore, the waiting period after placing intravenous infusion line lasted long, resulting in similar normal range cortisol levels in both groups or conditions immediately before the experimental intervention. The double-blind design further minimized influences of potentially confounding variables. Strict screening protocols and random assignments to 
groups or conditions also avoided significant differences in basal HPA axis activity at the morning of each measurement, which is a strong indication that experimental groups were matched even beyond their demographics. Importantly, effects of GC infusion were measured under resting conditions, i.e., a physiological baseline (Gusnard and Raichle, 2001), and therefore avoided any event-related (evoked) experimental stimulation that could cover-up or counteract GC actions. Noninvasive imaging methods, such as CASL, were used to avoid psychological stress from the application of tracer substances. In addition, the strength of CASL sequences, as well as variations in CASL perfusion measurements, is their ability to accurately assess absolute CNS perfusion during resting conditions, i.e., intrinsic, non-event-related activation, which distinguishes them from more commonly applied BOLD fMRI protocols (for advantages of resting state analyses, see Raichle and Snyder, 2007).

Study 1 established a rapid and bilateral perfusion decrease of $15.92 \mathrm{ml} / 100 \mathrm{~g} / \mathrm{min}$ in the thalamus within the first minutes after cortisol infusion (19\% decrease relative to the absolute average baseline). Study 2 replicated this effect in the thalamus during the first $7 \mathrm{~min}$ after infusion, resulting in a perfusion decrease of $13.45 \mathrm{ml} / 100 \mathrm{~g} / \mathrm{min}$ (or $17.9 \%$ relative to baseline). Study 2 could not replicate the perfusion effect in the second measurement block (10-17 min postinfusion). This discrepancy may be caused by the increased influence of random errors in Study 2 ( $n=9$ vs $n=26$ in Study 1). It is possible that GCs have additional, smaller effects that may include the caudate nucleus and the exploratory ROIs of Study 1 (Table 2). Importantly, the timeframes of the CBF effects that we found in both studies allow the exclusion of currently known genomic mechanisms of cortisol action and point toward nongenomic cortisol mechanisms as a source of these CBF effects.

Hemodynamic effects of exogenous GCs on the thalamus have not yet been reported in humans. The only study that reported rapid effects on the thalamus after GC infusion was by Ferris and Stolberg (2010), who described the existence of a BOLD effect during the first $5 \mathrm{~min}$ after GC infusion in rats. However, this effect was not further evaluated or discussed. Wang and colleagues (2005) described a perfusion increase in the thalamus of healthy adults after a psychological stress challenge (mental arithmetic task) that is likely to induce endogenous cortisol secretion (after 20-30 min) along with the activation of the autonomic nervous system and involvement of emotional and cognitive processes. In contrast, the cortisol infusion in our studies mimics just one aspect of a genuine psychophysiological stress response by addressing the action of the GC specifically.

Thalamic nuclei play a crucial role in the adaptation to challenges by modulating thalamocortical and corticocortical communication (Guillery and Sherman, 2002; Sherman and Guillery, 2002; Sherman, 2007; Theyel et al., 2010). The spatial resolution of our CBF images did not allow a reliable localization of functionally distinct thalamic nuclei. In general, however, thalamic functioning has been tied to processes of attention, consciousness, and arousal (Newman, 1995; Portas et al., 1998; Sarter et al., 2001), and sensorimotor integration and anticipation (Newman, 1995; Brunia, 1999). Thalamic functioning has been causally linked to EEG activity (Steriade et al., 1990; Lopes da Silva, 1991; Nunez et al., 2001; Hughes and Crunelli, 2005) and therefore constitutes a possible origin for GC-induced effects on EEG. Rather recently, a novel view has also been proposed in which the role of the thalamus does not end after sensory signals are transmitted to the cortex. Instead, the thalamus itself may play a crucial role in transmitting information between cortical areas or in coordinating the activity of different cortical areas (Sherman, 2001, 2007; Sherman and Guillery, 2002, 2006). A number of thalamic nuclei receive their driving input from the cortex and in turn may relay these signals to other cortical regions via ascending thalamocortical projections. Therefore, cortisol-induced changes in thalamic functioning are well posed to affect the activity of corticothalamocortical loops.

The EEG results of Study 3, rapid, widely distributed, and long-lasting changes in signal strength and map dissimilarity of EEG resting activity (Fig. 4), were established, which also have not previously been reported after cortisol infusion. Signal strength and map dissimilarity decreased in the cortisol compared with the placebo condition in all three postinfusion measurements (up to $27 \mathrm{~min}$ postinfusion). While it is theoretically possible that later effects ( $>20 \mathrm{~min})$ may in part be supplemented through genomic mechanisms, the early onset of the effects on EEG again implies the action of nongenomic mechanisms.

The cortisol-induced decrease in EEG signal strength suggests that cortisol acts on cortical functioning by dampening global brain activity patterns instead of influencing an isolated region or process. Cortical processing load that typically increases during a stressful situation [stressors are known to increase cognitive and emotional processing (Pruessner et al., 2010)] may be diminished by cortisol action, thereby protecting the brain as a whole from a state of processing overload and allowing adaptation to the challenge. A down-tuning of background processing may deliberate cognitive resources and ultimately improve the processing of potentially important and demand-specific sensory inputs (i.e., focus). Shifts of cognitive operating capacities may result in faster detection of stimulus-response contingencies, such as a recent report on trace eye-blink conditioning implies (Kuehl et al., 2010). Furthermore, it may result in a relatively enhanced (less inhibition) processing of startling danger signals (Richter et al., 2011). In addition, decreases in map dissimilarity in the cortisol condition may represent an enhanced spatial similarity of neuronal activity and indicate a more focused processing. Future studies may reach beyond our implemented exploratory design by focusing on relevant behavioral or physiological measurements that characterize the suggested GC-induced functional streamlining. For example, the down-tuning of background activation may improve signal-to-noise ratios for $\mathrm{N} 1$ or P300 components of the EEG. It also remains to be shown whether the cortisolinduced thalamic effects are indeed linked to the changes in signal strength and map dissimilarity, but the apparent implications for understanding normal (and eventually pathological) stress responses in humans makes this worth further investigations.

In summary, the observed GC-induced changes on EEG suggest a rapid, putative nongenomic, neuromodulatory effect that streamlines global neuronal functioning toward effective adaptation to stressors. Due to the time-restricted fast action of cortisol, we may reject the well known negative feedback action of the hormone-restoring homeostasis as an explanation for the global suppression effects of cortisol on the brain (de Kloet et al., 2005).

The physiological origins of the observed changes in CBF can be twofold. One, GCs may hypothetically act on the vascular resistance on the levels of arterioles, which would influence the postarteriole blood flow (Gros et al., 2007). Another explanation lies in the effect on the metabolism of cells: a GC-mediated decrease in neuronal activity would decrease metabolic needs and cause reduced CBF. The specific endocrine pathways by which GCs can rapidly and nongenomically influence such changes in neuronal activity are still unknown (for a general overview of possible pathways in animal models, see Groeneweg et al., 2011). 
The observed changes in EEG signal strength and map dissimilarity are related to changes in neuronal activity. Unfortunately, we cannot statistically link EEG effects to MRI-measured changes in the thalamus, since both measurements were assessed separately and in different participants. However, magnitude and direction of the correlational results of Figure 5 provide the principle requirements for a true relationship between cortisol, thalamic, and neuronal functioning, which would be in accordance with the above-mentioned novel view on corticothalamocortical loops. Simultaneous EEG-MRI measurements seem well suited to further investigate whether such a correlational link indeed exists or whether the induced changes in signal strength and map dissimilarity are linked to direct GC action on individual neurons or activity changes in brain areas other than the thalamus. Further investigations are especially important since map dissimilarity did not correlate with the acute cortisol levels to the same degree as signal strength, which indicates that there may be different effect mechanisms at play. In addition, further studies are required to extend our results, which are currently only valid under low cognitive load and, until shown otherwise, cannot be expanded for settings that exceed resting periods.

In conclusion, our data are the first to coherently suggest that a physiological concentration of cortisol profoundly affects functioning and perfusion of the human CNS in vivo by a rapid, nongenomic mechanism. The changes in neuronal functioning suggest that cortisol acts on the thalamic relay, thereby possibly suppressing processing of nonrelevant background information and allowing focus on task-specific sensory information to facilitate the adaptation to stress challenges.

\section{References}

Alsop DC, Detre JA (1996) Reduced transit-time sensitivity in noninvasive magnetic resonance imaging of human cerebral blood flow. J Cereb Blood Flow Metab 16:1236-1249.

Bell AJ, Sejnowski TJ (1995) An information-maximization approach to blind separation and blind deconvolution. Neural Comput 7:1129-1159.

Bloomfield P (1976) Fourier analysis of time series: an introduction. New York: Wiley.

Brunia CH (1999) Neural aspects of anticipatory behavior. Acta Psychol (Amst) 101:213-242.

Chatrian GE, Lettich E, Nelson PL (1988) Modified nomenclature for the "10\%" electrode system. J Clin Neurophysiol 5:183-186.

Cohen J (1988) Statistical power analysis for the behavioral sciences. Hilldale, New York: Erlbaum.

Coirini H, Marusic ET, De Nicola AF, Rainbow TC, McEwen BS (1983) Identification of mineralocorticoid binding sites in rat brain by competition studies and density gradient centrifugation. Neuroendocrinology 37:354-360.

Dallman MF (2005) Fast glucocorticoid actions on brain: back to the future. Front Neuroendocrinol 26:103-108.

Dedovic K, Duchesne A, Andrews J, Engert V, Pruessner JC (2009) The brain and the stress axis: the neural correlates of cortisol regulation in response to stress. Neuroimage 47:864-871.

De Kloet ER, Veldhuis HD, Wagenaars JL, Bergink EW (1984) Relative binding affinity of steroids for the corticosterone receptor system in rat hippocampus. J Steroid Biochem 21:173-178.

De Kloet ER, Vreugdenhil E, Oitzl MS, Joëls M (1998) Brain corticosteroid receptor balance in health and disease. Endocr Rev 19:269-301.

de Kloet ER, Joëls M, Holsboer F (2005) Stress and the brain: from adaptation to disease. Nat Rev Neurosci 6:463-475.

Dressendörfer RA, Kirschbaum C, Rohde W, Stahl F, Strasburger CJ (1992) Synthesis of a cortisol-biotin conjugate and evaluation as a tracer in an immunoassay for salivary cortisol measurement. J Steroid Biochem Mol Biol 43:683-692.

Dumermuth G, Molinari L (1987) Spectral analysis of the EEG: some fundamentals revisited and some open problems. Neuropsychobiology 17:85-99.
Dunn OJ (1961) Multiple comparison among means. J Am Stat Assoc 56:52-64.

Evanson NK, Herman JP, Sakai RR, Krause EG (2010) Nongenomic actions of adrenal steroids in the central nervous system. J Neuroendocrinol 22:846-861.

Ferris CF, Stolberg T (2010) Imaging the immediate non-genomic effects of stress hormone on brain activity. Psychoneuroendocrinology 35:5-14.

Friston KJ, Holmes AP, Worsley KJ, Poline JB, Frith CD, Frackowiak RS (1995) Statistical parametric maps in functional imaging: a general linear approach. Hum Brain Mapp 2:189-210.

Gasser T, Bächer P, Möcks J (1982) Transformations towards the normal distribution of broad band spectral parameters of the EEG. Electroencephalogr Clin Neurophysiol 53:119-124.

Groeneweg FL, Karst H, de Kloet ER, Joëls M (2011) Rapid non-genomic effects of corticosteroids and their role in the central stress response. J Endocrinol 209:153-167.

Gros R, Ding Q, Armstrong S, O’Neil C, Pickering JG, Feldman RD (2007) Rapid effects of aldosterone on clonal human vascular smooth muscle cells. Am J Physiol Cell Physiol 292:C788-C794.

Guillery RW, Sherman SM (2002) The thalamus as a monitor of motor outputs. Philos Trans R Soc Lond B Biol Sci 357:1809-1821.

Gusnard DA, Raichle ME (2001) Searching for a baseline: functional imaging and the resting human brain. Nat Rev Neurosci 2:685-694.

Hermes M, Hagemann D, Britz P, Lieser S, Rock J, Naumann E, Walter C (2007) Reproducibility of continuous arterial spin labeling perfusion MRI after 7 weeks. MAGMA 20:103-115.

Hermes M, Hagemann D, Britz P, Lieser S, Bertsch K, Naumann E, Walter C (2009) Latent state-trait structure of cerebral blood flow in a resting state. Biol Psychol 80:196-202.

Herscovitch P, Raichle ME (1985) What is the correct value for the brainblood partition coefficient for water? J Cereb Blood Flow Metab 5:65-69.

Holm S (1979) A simple sequentially rejective multiple test procedure. Scand J Stat 6:65-70.

Hughes SW, Crunelli V (2005) Thalamic mechanisms of EEG alpha rhythms and their pathological implications. Neuroscientist 11:357-372.

Jung TP, Makeig S, Humphries C, Lee TW, McKeown MJ, Iragui V, Sejnowski TJ (2000) Removing electroencephalographic artifacts by blind source separation. Psychophysiology 37:163-178.

Karst H, Berger S, Turiault M, Tronche F, Schütz G, Joëls M (2005) Mineralocorticoid receptors are indispensable for nongenomic modulation of hippocampal glutamate transmission by corticosterone. Proc Natl Acad Sci U S A 102:19204-19207.

Koenig T, Gianotti LR (2009) Scalp field maps and their characterization. In: Electrical neuroimaging (Michel CM, Koenig T, Brandeis D, Gianotti LRR, Wackermann J, eds), pp 25-47. Cambridge: Cambridge UP.

Kuehl LK, Lass-Hennemann J, Richter S, Blumenthal TD, Oitzl M, Schachinger H (2010) Accelerated trace eyeblink conditioning after cortisol IVinfusion. Neurobiol Learn Mem 94:547-553.

Lehmann D, Ozaki H, Pal I (1987) EEG alpha map series: brain micro-states by space-oriented adaptive segmentation. Electroencephalogr Clin Neurophysiol 67:271-288.

Lopes da Silva F (1991) Neural mechanisms underlying brain waves: from neural membranes to networks. Electroencephalogr Clin Neurophysiol 79:81-93.

Lovallo WR, Robinson JL, Glahn DC, Fox PT (2010) Acute effects of hydrocortisone on the human brain: an fMRI study. Psychoneuroendocrinology 35:15-20.

Makara GB, Haller J (2001) Non-genomic effects of glucocorticoids in the neural system: evidence, mechanisms and implications. Prog Neurobiol 65:367-390.

Makeig S, Bell AJ, Jung TP, Sejnowski TJ (1996) Independent component analysis of electroencephalographic data. In: Advances in neural information processing systems (Touretzky D, Mozer M, Hasselmo M, eds), pp 145-151. Cambridge: MIT.

Mikics E, Kruk MR, Haller J (2004) Genomic and non-genomic effects of glucocorticoids on aggressive behavior in male rats. Psychoneuroendocrinology 29:618-635.

Newman J (1995) Thalamic contributions to attention and consciousness. Conscious Cogn 4:172-193.

Nunez PL, Wingeier BM, Silberstein RB (2001) Spatial-temporal structures of human alpha rhythms: theory, microcurrent sources, multiscale mea- 
surements, and global binding of local networks. Hum Brain Mapp 13:125-164.

Portas CM, Rees G, Howseman AM, Josephs O, Turner R, Frith CD (1998) A specific role for the thalamus in mediating the interaction of attention and arousal in humans. J Neurosci 18:8979-8989.

Pruessner JC, Wolf OT, Hellhammer DH, Buske-Kirschbaum A, von Auer K, Jobst S, Kaspers F, Kirschbaum C (1997) Free cortisol levels after awakening: a reliable biological marker for the assessment of adrenocortical activity. Life Sci 61:2539-2549.

Pruessner JC, Kirschbaum C, Meinlschmid G, Hellhammer DH (2003) Two formulas for computation of the area under the curve represent measures of total hormone concentration versus time-dependent change. Psychoneuroendocrinology 28:916-931.

Pruessner JC, Dedovic K, Pruessner M, Lord C, Buss C, Collins L, Dagher A, Lupien SJ (2010) Stress regulation in the central nervous system: evidence from structural and functional neuroimaging studies in human populations: 2008 Curt Richter Award winner. Psychoneuroendocrinology 35:179-191.

Raichle ME, Snyder AZ (2007) A default mode of brain function: a brief history of an evolving idea. Neuroimage 37:1083-1090; discussion 1097-1099.

Richter S, Schulz A, Zech CM, Oitzl MS, Daskalakis NP, Blumenthal TD, Schächinger H (2011) Cortisol rapidly disrupts prepulse inhibition in healthy men. Psychoneuroendocrinology 36:109-114.

Roberts DA, Rizi R, Lenkinski RE, Leigh JS Jr (1996) Magnetic resonance imaging of the brain: blood partition coefficient for water: application to spin-tagging measurement of perfusion. J Magn Reson Imaging 6:363-366.

Sánchez MM, Young LJ, Plotsky PM, Insel TR (2000) Distribution of corticosteroid receptors in the rhesus brain: relative absence of glucocorticoid receptors in the hippocampal formation. J Neurosci 20:4657-4668.

Sarter M, Givens B, Bruno JP (2001) The cognitive neuroscience of sustained attention: where top-down meets bottom-up. Brain Res Brain Res Rev 35:146-160.

Sherman SM (2001) Tonic and burst firing: dual modes of thalamocortical relay. Trends Neurosci 24:122-126.
Sherman SM (2007) The thalamus is more than just a relay. Curr Opin Neurobiol 17:417-422.

Sherman SM, Guillery RW (2002) The role of the thalamus in the flow of information to the cortex. Philos Trans R Soc Lond B Biol Sci 357: 1695-1708.

Sherman SM, Guillery RW (2006) Exploring the thalamus and its role in cortical function, 2nd edition. Cambridge, MA: MIT.

Steriade M, Gloor P, Llinás RR, Lopes de Silva FH, Mesulam MM (1990) Report of IFCN committee on basic mechanisms: basic mechanisms of cerebral rhythmic activities. Electroencephalogr Clin Neurophysiol $76: 481-508$

Stevens J (1996) Applied multivariate statistics for the social sciences, 3rd edition. Hillsdale, NJ: Erlbaum.

Sutanto W, van Eekelen JA, Reul JM, de Kloet ER (1988) Species-specific topography of corticosteroid receptor types in rat and hamster brain. Neuroendocrinology 47:398-404.

Theyel BB, Llano DA, Sherman SM (2010) The corticothalamocortical circuit drives higher-order cortex in the mouse. Nat Neurosci 13:84-88.

Tzourio-Mazoyer N, Landeau B, Papathanassiou D, Crivello F, Etard O, Delcroix N, Mazoyer B, Joliot M (2002) Automated anatomical labeling of activations in SPM using a macroscopic anatomical parcellation of the MNI MRI single-subject brain. Neuroimage 15:273-289.

Ulrich-Lai YM, Herman JP (2009) Neural regulation of endocrine and autonomic stress responses. Nat Rev Neurosci 10:397-409.

Walter B, Blecker C, Kirsch P, Sammer G, Schienle A, Stark R, Vaitl D (2003) MARINA: an easy to use tool for creation of masks for region of interest analyses. Paper presented at 9th International Conference on Functional Mapping of the Human Brain, New York, June 19-22.

Wang J, Rao H, Wetmore GS, Furlan PM, Korczykowski M, Dinges DF, Detre JA (2005) Perfusion functional MRI reveals cerebral blood flow pattern under psychological stress. Proc Natl Acad Sci U S A 102:17804-17809.

Wang Z, Aguirre GK, Rao H, Wang J, Fernández-Seara MA, Childress AR, Detre JA (2008) Empirical optimization of ASL data analysis using an ASL data processing toolbox: ASLtbx. Magn Reson Imaging 26:261-269.

Wilhelm I, Born J, Kudielka BM, Schlotz W, Wüst S (2007) Is the cortisol awakening rise a response to awakening? Psychoneuroendocrinology 32: $358-366$. 